\section{A Remarkable Pattern of Subcortical Vessel Wall Enhancement in Granulomatous Angiitis of the Central Nervous System}

To the Editor:

Primary angiitis of the central nervous system (PACNS) is an idiopathic disorder. Clinical as well as radiological characteristics are nonspecific and heterogenic, making diagnosis delicate. We report an unusual pattern of extensive radial enhancement of the deep cerebral vasculature on magnetic resonance imaging (MRI) in a previously healthy 64-year-old woman.

Our patient presented with a 6-month history of seizures, weight loss, difficulty walking, and bilateral deterioration of vision. She and her partner had noticed progressive cognitive decline with decreased mental speed and lack of initiative. She scored 29/30 on the Mini Mental State Examination with remarkable mental slowness. Visual acuity was 20/40 (right) and 20/32 (left) with bilateral optic atrophy. She had asymmetrical parkinsonism with myoclonus of both hands and presence of primitive reflexes.

MRI of the brain demonstrated symmetrical white matter abnormalities (Figure 1A) with remarkably extensive enhancement after gadolinium in a radial pattern, following the deep cerebral vasculature (Figures 1B, 1C). Cerebrospinal fluid (CSF) showed a lymphocytic pleiocytosis $(47$ cells $/ \mu l)$,

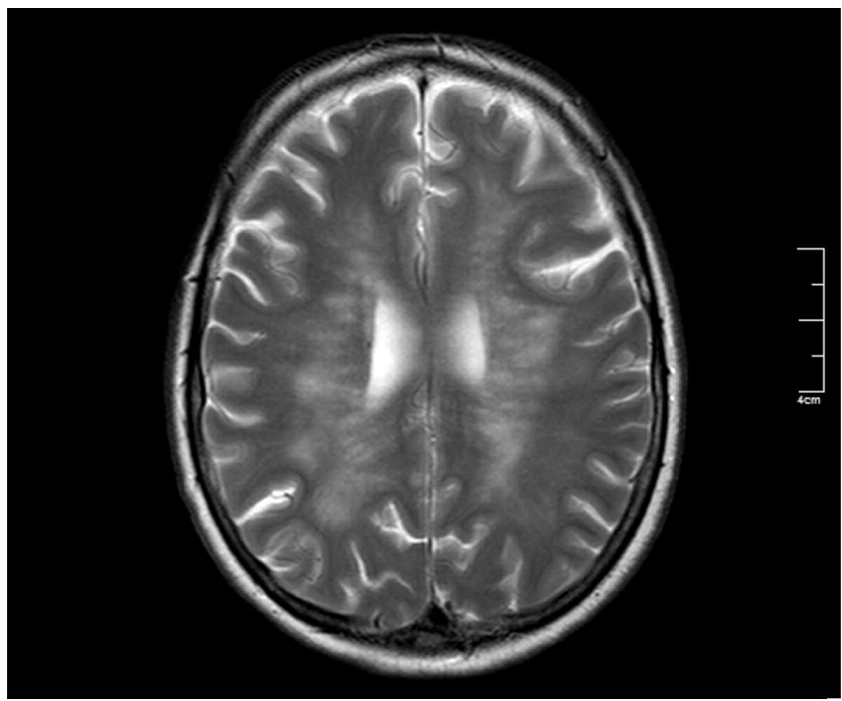

\section{A}

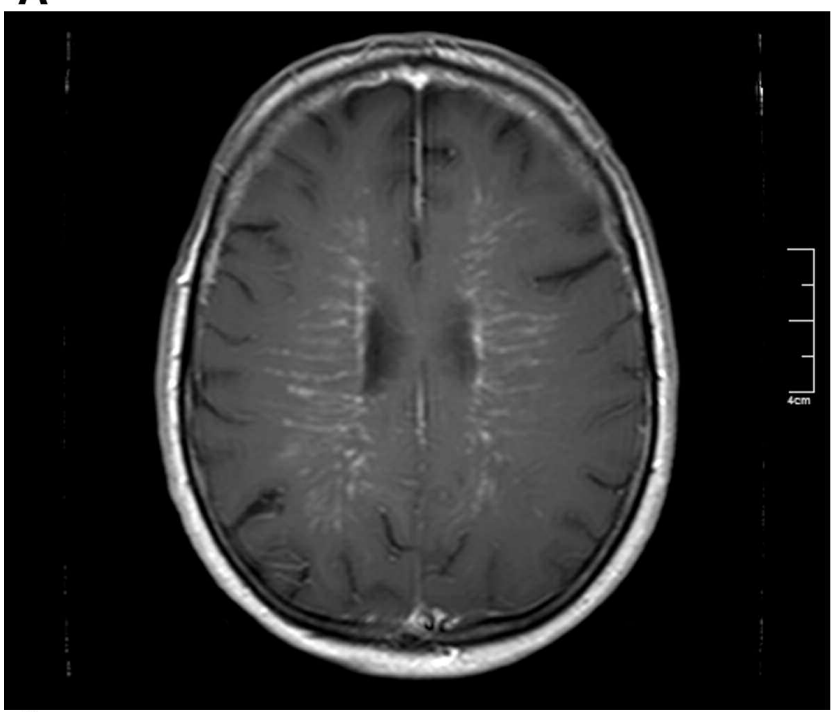

C elevated protein $(1.62 \mathrm{~g} / \mathrm{dl})$, and normal glucose concentration $(50 \mathrm{mg} / \mathrm{dl})$. Serological tests for Treponema pallidum, Borrelia burgdorferi, Mycobacterium tuberculosis, and human immunodeficiency virus were all negative as were microbiological, virological, pathological, and hematological CSF analyses. Laboratory assessment of blood and urine for rheumatic disease, paraneoplastic disorders, and sarcoidosis was negative. A total-body positron emission tomography scan was normal.

Because of the differential diagnostic consideration of a diffuse intravascular lymphoma, a stereotactic brain biopsy was performed. This revealed a microangiopathy with angiocentric granulomas (Figures 2A, 2B). Immunophenotyping showed no monoclonal lymphocyte populations. Immunohistochemistry on $\beta$-amyloid and microbiological stainings were all negative. She was diagnosed with idiopathic granulomatous angiitis of the CNS.

Treatment was initiated with intravenous methylprednisolone $1 \mathrm{~g} / \mathrm{day}$ for 3 days and oral prednisolone $20 \mathrm{mg}$ /day, with slow tapering of dosage contiguously. This prompted dramatic clinical improvement within weeks. At the 1-year followup, neurological examination revealed only subtle parkinsonism, with no relapses to date.

PACNS is a rare disorder of unknown etiology. Its exact incidence is unknown ${ }^{1}$. Diagnostic criteria require presence of an otherwise unex-

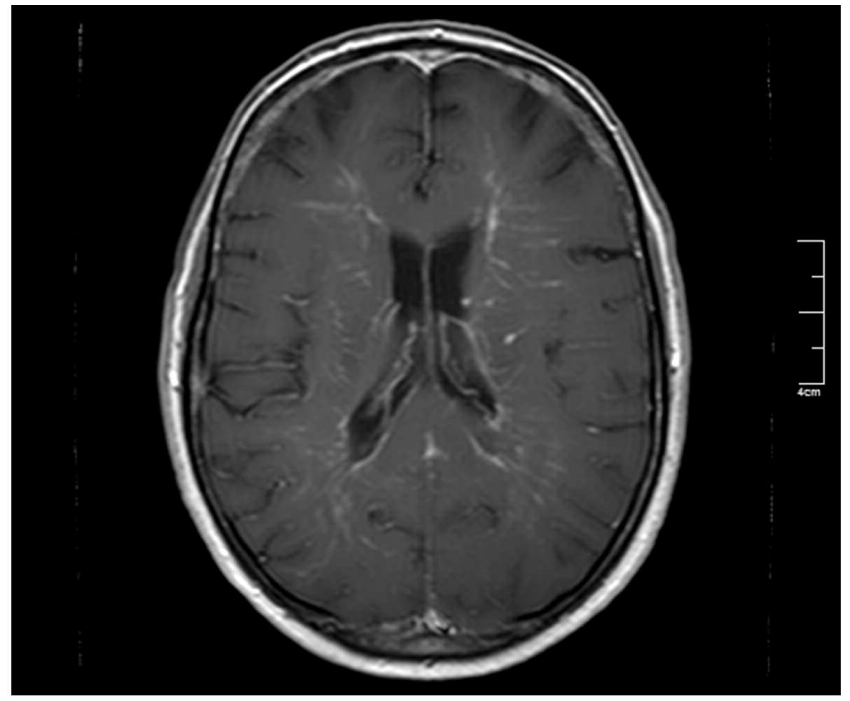

B

Figure 1. Magnetic resonance imaging of the brain showing symmetrica white matter lesions on axial T2-weighted scan (A). After gadolinium, axial T1-weighted images show extensive subcortical enhancement following the deep cerebral vasculature $(\mathrm{B}, \mathrm{C})$ 

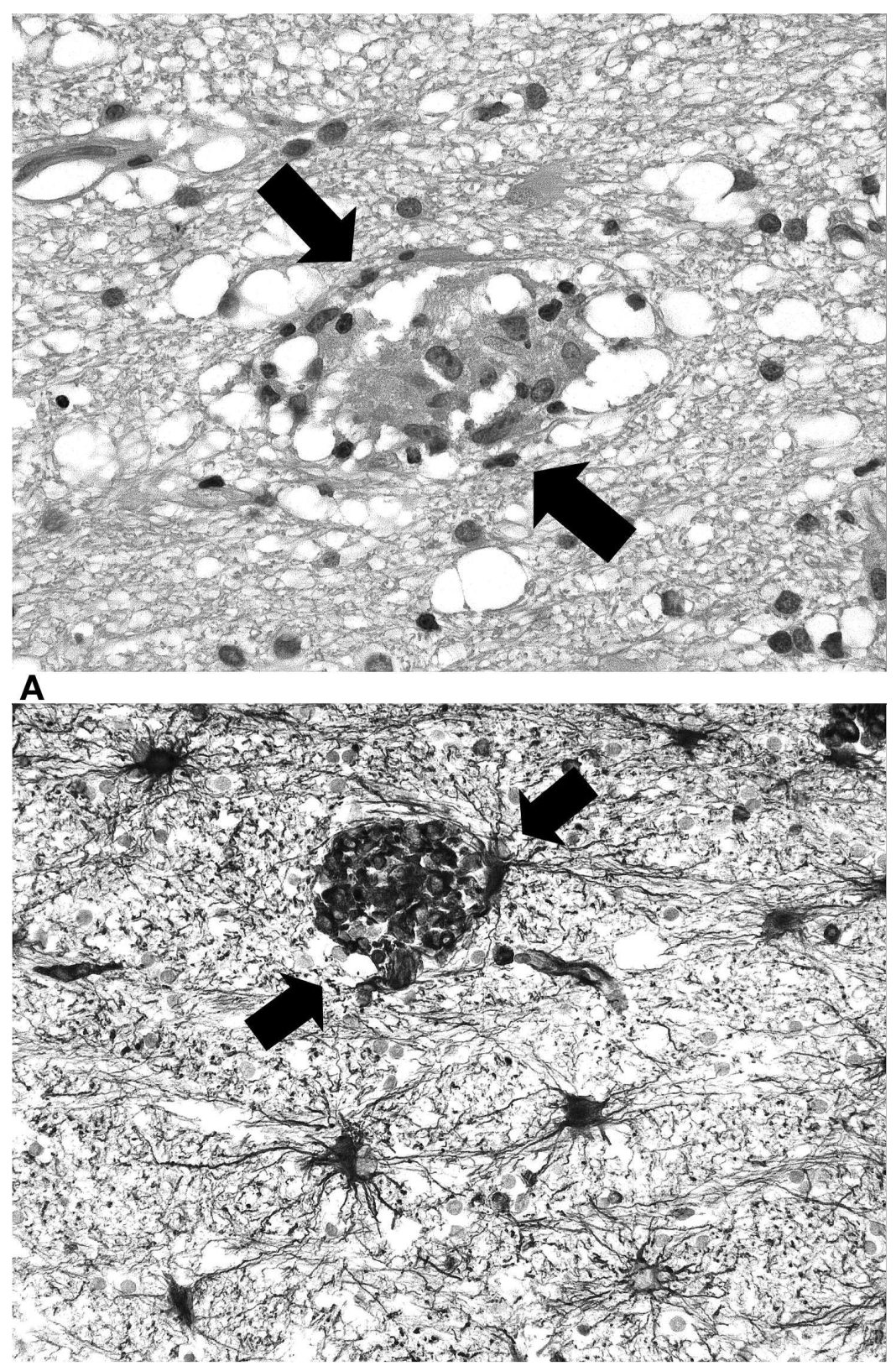

B

Figure 2. H\&E stained section of right frontal subcortical brain tissue revealing a focal granulomatous inflammatory lesion with microvascular destruction within the cerebral white matter (A, arrows). A vimentin stained section of the biopsy shows a granuloma with a cluster of histiocytes within intense gliotic white matter (B, arrows).

plained neurological or psychiatric deficit in concurrence with supporting angiographic or neuropathological features and absence of systemic vasculitis or mimicking conditions ${ }^{2}$. Clinical characteristics are nonspecific and show marked heterogeneity. Most patients present with subacute development of headache, focal cerebral ischemia affecting multiple vascular territories, and aseptic meningitis ${ }^{3,4}$. A minority has a more protracted clinical course with symptoms of chronic meningitis preceding diagnosis as much as 5 years $^{4}$. As in our case, these patients have been neuropathologically diagnosed with the subtype of granulomatous angiitis of the CNS (GACNS) ${ }^{1}$.

Abnormalities on MRI are present in $90 \%-100 \%$ of patients, but simi- lar to clinical characteristics, the abnormalities are both highly variable and nonspecific ${ }^{1}$. Cerebral infarcts are most common and occur in up to $54 \%$ of patients. Infarcts are often multiple and can have a large-vessel as well as small-vessel distribution ${ }^{4}$. Other associated abnormalities are nonspecific white matter lesions, intracerebral hemorrhage, mass lesions, and contrast-enhancing intracerebral or leptomeningeal lesions ${ }^{1,4}$. Vessel wall enhancement has been suggested as a diagnostic sign with high sensitivity in a recent retrospective case series ${ }^{5}$. In this cohort, abnormalities were predominantly in a large-vessel distribution. To our knowledge, the diffuse and extensive contrast enhancement confined to the deep cerebral vasculature that was demonstrated in our case has not been described previously.

Personal non-commercial use only. The Journal of Rheumatology Copyright @ 2012 . All rights reserved. 
Because of the heterogenic presentation of the disorder, recognition of associated neuroradiological patterns is of key importance in raising clinical suspicion and driving diagnostic investigations. Therapeutic relevance is emphasized by the dramatic clinical improvement this patient showed on steroid treatment.

NIELS SCHOENMAKER, MD, Department of Neurology; MENNO R. GERMANS, MD, Department of Neurosurgery; DIRK TROOST, MD, PhD, Department of Pathology; EDO RICHARD, MD, PhD, Department of Neurology, Academic Medical Centre, Amsterdam, The Netherlands. Address correspondence to Dr. N. Schoenmaker, Department of Neurology, Academic Medical Centre, PO Box 22660, 1100 DD Amsterdam, The Netherlands. E-mail: n.schoenmaker@amc.uva.nl

\section{REFERENCES}

1. Hajj-Ali RA, Singhal AB, Benseler S, Molloy E, Calabrese LH. Primary angiitis of the CNS. Lancet Neurol 2011;10:561-72.

2. Calabrese LH, Mallek JA. Primary angiitis of the central nervous system: Report of 8 new cases, review of the literature, and proposal for diagnostic criteria. Medicine 1988;67:20-39.

3. Salvarani C, Brown RD Jr, Calamia KT, Christianson TJ, Weigand SD, Miller DV, et al. Primary central nervous system vasculitis: analysis of 101 patients. Ann Neurol 2007;62:442-51.

4. Salvarani C, Brown RD Jr, Calamia KT, Christianson TJ, Huston J 3rd, Meschia JF, et al. Rapidly progressive primary central nervous system vasculitis. Rheumatology 2011;50:349-58.

5. Küker W, Gaertner S, Nagele T, Dopfer C, Schoning M, Fiehler J, et al. Vessel wall contrast enhancement: A diagnostic sign of cerebral vasculitis. Cerebrovasc Dis 2008;26:23-9. 